\title{
Biological and Psychological Stress Correlates Are Linked to Glucose Metabolism, Obesity, and Gender Roles in Women
}

\author{
Alexander Kautzky ${ }^{a} \quad$ Kathrin Heneis $^{b, c}$ Karin Stengg ${ }^{d}$ Sabine Fröhlich ${ }^{d}$ \\ Alexandra Kautzky-Willer ${ }^{\mathrm{b}, \mathrm{c}}$ \\ aDepartment of Psychiatry and Psychotherapy, Medical University of Vienna, Vienna, Austria; ${ }^{\mathrm{b}}$ Division of \\ Endocrinology and Metabolism, Department of Internal Medicine III, Medical University of Vienna, Vienna, Austria; \\ 'Gender Institute, Gars am Kamp, Austria; d"La Pura" Womens Health Resort, Gars am Kamp, Austria
}

\section{Keywords}

Stress · Obesity $\cdot$ Insulin trafficking

\begin{abstract}
Objectives: Psychological stress affects central as well as peripheral metabolism and hormone trafficking via the hypothalamic-pituitary-adrenal axis. Stress thereby plays a decisive role in the etiology and progression of overweight and obesity, leading to several chronic diseases, such as diabetes, and mental health disorders. The interplay of biological and psychometric correlates of stress, anthropometric, immunological, and metabolic parameters and psychosocial factors such as gender roles, however, remains poorly understood. Methods: In this exploratory study, 43 healthy women were assessed for glucose metabolism by an oral glucose tolerance test and computation of functional parameters for insulin secretion, sensitivity, and resistance. Further, the fatty liver index (FLI) and anthropometric parameters body mass index (BMI), waist-to-hip ratio, body fat, and lean mass were assessed. Psychological stress assessment included the "Brief Symptom Inventory" (BSI), the "Burnout Dimensions Inventory" (BODI), and Perceived Stress Scale (PSS). Biologi-
\end{abstract}

cal stress response was evaluated with heart rate variability and cortisol levels. Finally, gender role self-identification was assessed with the "Bem Sex-Role Inventory" (BSRI). Generalized linear models were computed for exploratory association with psychometric outcome. Uncorrected $p$ values are reported. Results: Burnout and PSS scores were associated with insulin secretion, sputum cortisol, thyroid-stimulating hormone, anthropometric measures, and gender role. BSI ratings for psychiatric symptom dimensions were associated with insulin resistance, sex hormones, anthropometric measures, and gender role. Female self-identification was associated with higher BMI as well as body fat and a higher FLI. Conclusions: Considering the increased risk of unfavorable metabolic, cardiovascular, and also mental health outcome in obese women, a higher BMI in women with predominant female gender self-identification may be relevant for clinical risk assessment. The broad range of interacting biological, psychological, and gender-related parameters calls for an integrative management of both mental and endocrinological health. However, the exploratory nature of the study requires replication in larger samples before definite conclusion can be drawn.

(c) 2021 The Author(s)

Published by S. Karger AG, Basel karger@karger.com www.karger.com/nen

Karger $\stackrel{\text { ' }}{5}$

BOPEN ACCESS
(C) 2021 The Author(s)

Published by S. Karger AG, Basel

This is an Open Access article licensed under the Creative Commons Attribution-NonCommercial-4.0 International License (CC BY-NC) (http://www.karger.com/Services/OpenAccessLicense), applicable to the online version of the article only. Usage and distribution for commercial purposes requires written permission.
Correspondence to:

Alexander Kautzky, alexander.kautzky@meduniwien.ac.at 


\section{Introduction}

Disorders rooted in both, mental health and metabolism, have been on a steady rise for decades $[1,2]$. Affective disorders, most commonly the major depressive disorder, constitute a substantial share in global disability and disease burden and affect an estimated $5-10 \%$ of the global population [3]. On the other hand, about a fifth of the world population have a body mass index (BMI) of over 30 , indicating obesity and resulting in high rates of metabolic disorders such as diabetes mellitus (DM) [4]. Currently, an estimated $9 \%$ of people worldwide suffer from DM type 2, a chronic disorder oftentimes leading to disability and premature death due to severe sequelae $[5,6]$. Both affective disorders and DM 2 are alarmingly widespread disorders challenging day-to-day life and bringing along substantial lifetime morbidity and mortality. Furthermore, psychoeducation and treatment adherence are essential for reaching satisfactory disease outcome for patients as well as doctors in both disorders $[7,8]$.

Despite the high numbers of patients treated for these disorders, the underlying etiopathological mechanisms are still insufficiently understood. Interestingly, being diagnosed with an affective disorder, especially major depressive disorder, multiplies the risk for developing diabetes, and vice versa [9]. This can partly be explained by the resounding psychosocial impact of these disorders. On the one hand, being diagnosed with any chronic disorder and facing a lifetime of health self-management and medical treatment is known to stress one's mental health. On the other hand, behavioral changes mediated by reduced energy, drive, and ability of self-care that occur in many mental health disorders naturally increase the risk for metabolic syndrome and DM 2. However, there are also biological pathways putatively linking mental health disorders to metabolic syndrome and DM 2. Among these, the immune system and subclinical inflammation attract attention. Thereby, chronic psychological stress may lead to a vulnerability for both disorders by interference with the hypothalamic-pituitary-adrenal axis, a main hub for neuroendocrine processing [10]. Alterations in cortisol trafficking are a well-published finding in both disorders [11], similar to elevated inflammation markers such as the $\mathrm{C}$-reactive protein (CRP) and interleukins $[12,13]$. Further elucidating the role of psychological stress, the biological stress response was measured with electrocardiogram and found to be different between patients with anxiety disorders and healthy individuals [14]. A high heart rate variability (HRV), describing the variation of physiological time intervals between
2 heartbeats was thereby indicative for physiological stress reaction and highlighted as a protective marker for cardiovascular events $[15,16]$. Consequently, biofeedback training of HRV was suggested to alleviate stress and protect from negative cardiovascular as well as mental health outcome $[17,18]$.

Nevertheless, the role of stress and the hypothalamicpituitary-adrenal axis in the pathophysiology of affective and anxiety as well as metabolic disorders remains shrouded. Considering that all these diseases are chronic and significantly impact everyday life, they bring along a need for continuous medication and thus side effects. Further, they oftentimes spark a high lifetime risk for severe comorbidities. To elucidate the complex link between mental and metabolic health requires consideration of many heterogenous factors. Putatively, there is also a moderating effect of biological sex and gender roles on this pattern as women are more likely to develop many psychiatric disorders than men [19].

Following these thoughts, stress may lead to an immuno-metabolic state that produces a sex-sensitive phenotype, displaying both metabolic and neuropsychiatric deflections early on. Consequently, the aim of this study was to substantiate the biological and psychosocial underpinnings of mental health by assessing metabolic parameters, biological as well as psychological correlates of stress and gender roles in healthy women not yet diagnosed with either depression or diabetes, but self-perceived stress and overweight or obesity.

\section{Methods}

Sample

Forty-three women (mean age $53.42 \pm 11.7$ ) were recruited between September 2019 and February 2020 at the VAMED Gender Institute (https://www.vamed.com/en/company/gender-institute/), a cooperating center of the "Gender Medicine Unit" of the University Clinic for Internal Medicine III, Department for Endocrinology and Metabolism. Women who were interested in reducing stress and weight by a combined psychological and dietoriented intervention applied for the study and were assessed by trained clinicians and psychologists during a stay at the "la pura women's health resort" (https://www.lapura.at/). Women had to be older than 18 years, not currently pregnant, and free of any acute or a severe chronic illness. Written informed consent after detailed oral information concerning all study procedures was mandatory for all subjects. All study procedures were approved by the responsible Ethics Committee.

\section{Measures}

All subjects underwent a physical examination for registration of anthropometric parameters: BMI, waist and hip circumference, waist-to-height ratio, and waist-to-hip ratio (WHR). Lean mass, 
Table 1. Sample characteristics for all predictors and outcome variables with means, standard deviations, and number of data entries

\begin{tabular}{|c|c|c|}
\hline & Mean \pm SD & $N$ \\
\hline \multicolumn{3}{|c|}{ Anthropometric parameters } \\
\hline Age, years & $53.42 \pm 11.7$ & \multirow{5}{*}{43} \\
\hline $\mathrm{BMI}$ & $27.56 \pm 5.77$ & \\
\hline Waist circumference & $97.99 \pm 14.19$ & \\
\hline Hip circumference & $117.44 \pm 19.21$ & \\
\hline WHR & $0.84 \pm 0.09$ & \\
\hline Body fat & $27.66 \pm 13.07$ & \multirow{2}{*}{31} \\
\hline Lean mass & $47.65 \pm 5.44$ & \\
\hline \multicolumn{3}{|l|}{ Metabolic parameters } \\
\hline HbA1c & $5.3 \pm 0.3$ & \multirow{3}{*}{43} \\
\hline OGTT baseline & $85.97 \pm 9.32$ & \\
\hline OGTT $120 \mathrm{~min}$ & $109.69 \pm 32.37$ & \\
\hline Matsuda Index & $6.02 \pm 3$ & 40 \\
\hline HOMA-IR & $1.83 \pm 1.03$ & 42 \\
\hline OGIS & $443.97 \pm 54.62$ & 33 \\
\hline IGI & $1.12 \pm 1.06$ & 31 \\
\hline Disposition index & $6.12 \pm 7.74$ & 30 \\
\hline FLI & $41.22 \pm 28.94$ & 43 \\
\hline \multicolumn{3}{|c|}{ Immuno-inflammatory parameters } \\
\hline CRP & $0.36 \pm 0.42$ & \multirow{3}{*}{43} \\
\hline Cortisol (serum) & $15.71 \pm 4.83$ & \\
\hline Cortisol (sputum) & $0.53 \pm 0.3$ & \\
\hline \multicolumn{3}{|l|}{ Hormones } \\
\hline Estradiol & $49.58 \pm 70.09$ & \multirow{4}{*}{43} \\
\hline Testosterone & $0.22 \pm 0.16$ & \\
\hline FSH & $51.33 \pm 36.37$ & \\
\hline $\mathrm{LH}$ & $29.64 \pm 19.33$ & \\
\hline $\mathrm{TSH}$ & $1.92 \pm 1.55$ & 22 \\
\hline Prolactin & $15.63 \pm 9.52$ & \\
\hline
\end{tabular}

body fat, and the phase angle were measured by bioelectrical impedance analysis.

Further, all subjects underwent an oral glucose tolerance test, and functional parameters were computed based on venous plasma insulin and glucose levels from baseline and 30, 60, 90, and 120 min after application of 75 g glucose. Therefore, the homeostasis model was used for assessment of insulin resistance (HOMA-IR) [20] and the Matsuda Index and oral glucose insulin sensitivity (OGIS) were calculated for assessment of insulin sensitivity [21, 22]. Insulin secretion was assessed by the insulinogenic index (IGI) [23] and beta-cell function by the disposition index (IGI*ISI) [24]. Further, the glycated hemoglobin (HbA1c) was measured for categorization of (pre)diabetes.

Laboratory assessment included the inflammation and immunology parameters CRP, serum cortisol, and sputum cortisol measured at 08:00 a.m., as well as lipid and liver function parameters (HDL, LDL, triglycerides, OGT, and GGT). Further, thyroid-stimulating hormone (TSH), prolactin, and the sexual hormones follicle-stimulating hormone (FSH), luteinizing hormone (LH), estradiol, and testosterone, were analyzed. For assessment of liver function, the fatty liver index (FLI) was computed from BMI, waist circumference, serum triglycerides, and serum GGT [25].

\begin{tabular}{l} 
Gender role scores \\
Male self-identification \\
Female self-identification \\
Neutral self-identification \\
\hline Psychological parameters \\
HRV baseline \\
HRV stress \\
HRV relaxation \\
PSS \\
Stress (family life) \\
Stress (work life) \\
Stress (self-oriented) \\
BSI total score \\
Anxiety (BSI) \\
Depression (BSI) \\
Somatization (BSI) \\
Paranoia (BSI) \\
Psychoticism (BSI) \\
Interpersonal sensitivity (BSI) \\
Aggression (BSI) \\
Compulsion (BSI) \\
BODI 1 \\
BODI 2 \\
BODI 3 \\
BODI 4
\end{tabular}

Mean \pm SD

N

Gender role scores

Gelf-identification

$4.88 \pm 0.69$

$4.64 \pm 0.69$

43

$4.34 \pm 0.39$

Psychological parameters

HRV baseline

$13.32 \pm 12.74$

$16.76 \pm 12.85$

$16.115 \pm 11.26$

$15.59 \pm 5.32 \quad 27$

$45.74 \pm 30.53 \quad 39$

$49.95 \pm 35.01 \quad 38$

$55.97 \pm 24.58 \quad 39$

$56.42 \pm 10.25$

$51.79 \pm 7.92$

$54.69 \pm 9.32$

$56.14 \pm 10.17$

$54.41 \pm 8.81$

$53.37 \pm 7.43$

$54.91 \pm 9.53$

$52.58 \pm 10.82$

$55.02 \pm 10.11$

$31.35 \pm 17.97$

$58.28 \pm 31.29$

$34.82 \pm 22.96$

37

$45.21 \pm 39.47$

OGTT, oral glucose tolerance test; FLI, fatty liver index; WHR, waist-to-hip ratio; BODI, Burnout Dimensions Inventory; PSS, Perceived Stress Scale; HRV, heart rate variability; TSH, thyroidstimulating hormone; CRP, C-reactive protein; HOMA-IR, homeostasis model was used for assessment of insulin resistance; OGIS, oral glucose insulin sensitivity; IGI, insulinogenic index; $\mathrm{FSH}$, follicle-stimulating hormone; $\mathrm{LH}$, luteinizing hormone.

In addition, the HRV was measured in all subjects by biofeedback assessment using the NeXus 10 from MindMedia (www. mindmedia.com/de/produkte/nexus-10-mkii/). HRV was registered at baseline as well as during consecutive stress and relaxation paradigms.

Finally, stress and mental health were assessed by a range of psychological questionnaires. These included the Brief Symptom Inventory (BSI), consistent of subscores for items paranoia, psychoticism, interpersonal sensitivity, anxiety, somatization, aggression, depression, and compulsion, as well as a global score [26]. Further, the "Burnout Dimensions Inventory" (BODI) was applied [27], composed of 40 questions that sum up to 4 items describing reduced resilience (BODI 1), reduced ability of distancing (BODI 2), depression (BODI 3), and dysfunctional compensation (BODI 4). Stress was also evaluated by the Perceived Stress Scale (PSS [28]), providing scores for the subitems of work, family, and selforiented stress as well as a global score (PSSI). Finally, gender role self-identification was assessed by the "Bem Sex-Role Inventory" (BSRI), measuring male, female, and neutral self-identification scores [29]. 

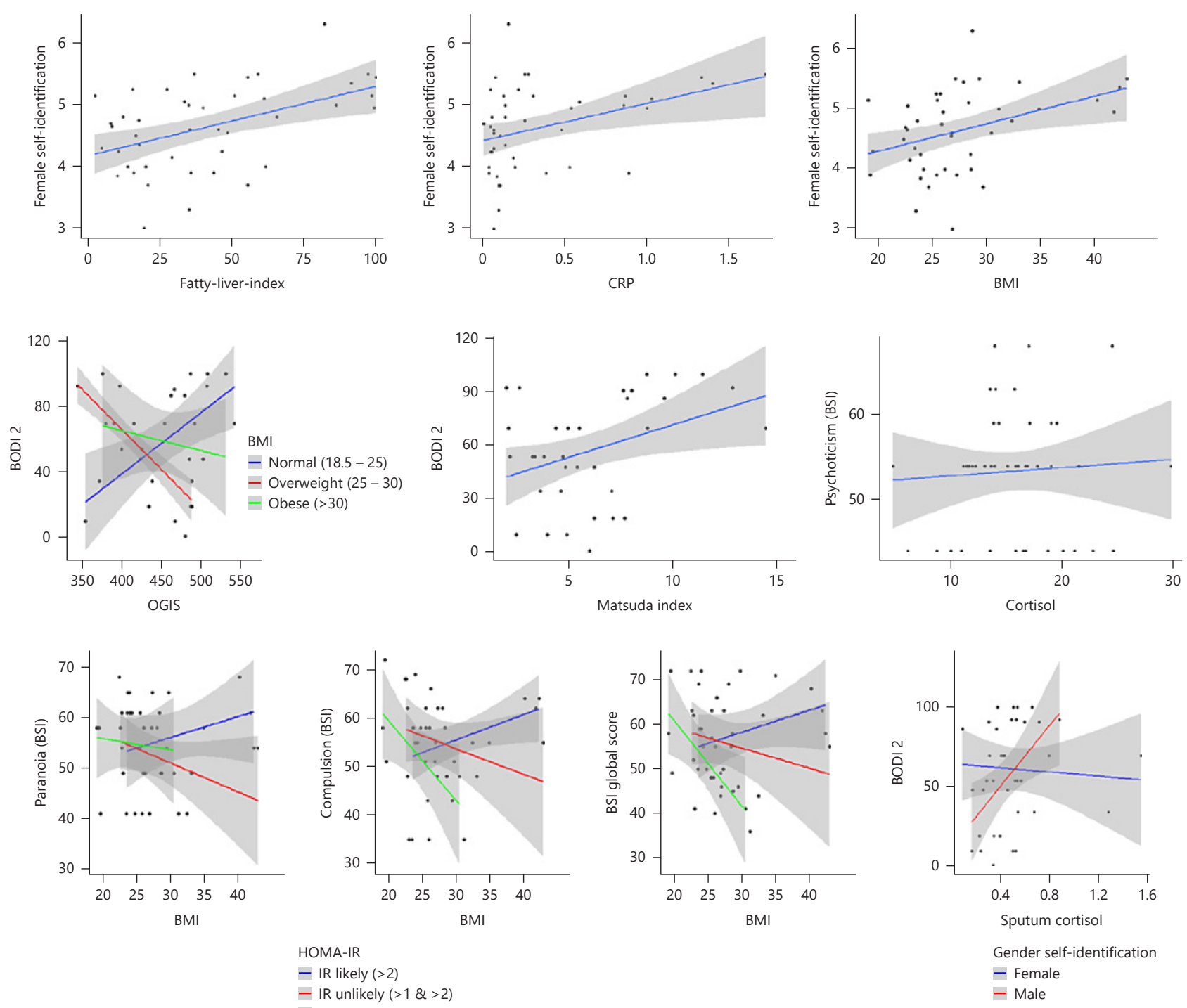

$$
\begin{aligned}
& \text { HOMA-IR } \\
& \text { - IR likely }(>2) \\
& \text { - IR unlikely }(>1 \&>2) \\
& \text { - No IR }(<1)
\end{aligned}
$$

Fig. 1. Main and interaction effects from the GLMs for metabolic and inflammatory parameters. Only significant $(p<0.05)$ effects are depicted. For illustration of interaction effects of 2 continuous variables, HOMA-IR was divided into a factor with 3 levels, indicating the likelihood of insulin presence. HOMA-IR, homeostasis model was used for assessment of insulin resistance; GLM, generalized linear model.

\section{Statistical Analyses}

Analyses were computed with statistical software "R" (www.rproject.org/). Generalized linear models (GLM) were applied to assess the effects of (1) BMI and insulin function parameters (Matsuda Index, OGIS, HOMA-IR, IGI, and disposition index); (2) metabolic (FLI) and anthropometric parameters (WHR, body fat, lean mass, and phase angle); (3) inflammation and immunology parameters (serum and sputum cortisol and CRP); (4) hormones (TSH, LH, FSH, estradiol, testosterone, and prolactin); and (5) biofeedback HRV parameters (baseline HRV, stress HRV, and relaxation HRV), on psychometric outcome parameters (BSI items
1-9 for psychiatric symptom categories depression, anxiety, phobic anxiety, interpersonal sensitivity, somatization, aggression, compulsion, paranoia, and psychoticism as well as the global severity index (GSI) score; PSS total score; BODI items 1-4, BODI self-rating for stress in work and family life as well as self-oriented stress) and render roles (BSRI scores for male, female, and neutral gender role self-identification).

All models, except those for gender role self-identification, included a binomial variable for predominant gender role (female or male), and 2- as well as three-way interaction effects. Deviance tables were computed with the ANOVA function for GLM, and 
nonsignificant interaction effects were dropped. All analyses were considered exploratory, and so, a $p$ value below 0.05 was regarded significant, and no correction for multiple testing was applied.

For all models, Cook's distance (CD) was computed to identify patients who significantly influence the performance of the regression models. Therefore, following a general rule, values $>3$ times the mean CD were considered significant [30]. The respective observations were checked for extreme values, defined by a value $>1.5$ times the interquartile distance. Models were rerun after capping of extreme values to the 90th percentile of the distribution of the respective parameter and results compared to the original models.

\section{Results}

A summary characterization of the sample can be found in Table 1. Overall, 35\% of the women scored a BMI between 18.5 and $25 \mathrm{~kg} / \mathrm{m}^{2}$, while $44 \%$ were overweight (BMI 25-30 kg/m²), 12\% showed obesity I' (BMI $30-35 \mathrm{~kg} / \mathrm{m}^{2}$ ), and 9\% obesity grade III $^{\circ}$ (BMI $40-45 \mathrm{~kg} /$ $\mathrm{m}^{2}$ ). Metabolic syndrome was present in $7 \%$ of the women according to the definition of the world diabetes federation [31].

All subjects showed an HbA1c below 6.5\% and fasting glucose below $126 \mathrm{mg} / \mathrm{dL}$, indicating absence of diabetes. A prediabetic state was found in 3 women $(\mathrm{HbAlc}>5.7)$. Possible insulin resistance, indicated by a HOMA-IR above 2 , was found in $36 \%$ women, and insulin secretion was reduced in 4 women, indicated by an IGI of $<0.4$. The disposition index was normal $(>1)$ in all subjects, displaying sufficient $\beta$-cell function. An increased risk for having a fatty liver, according to an FLI $>30$ but $<60$, was found in $35 \%$ and a likely fatty liver, shown by an FLI $>60$, in $23 \%$ of the women.

At least moderate self-perceived stress (PSSI $\geq 14$ ) was present in $67 \%$ of women $(15.59 \pm 5.32)$. Clinically relevant scores of the GSI $(\geq 63)$ of the BSI were observed in $32.5 \%$ of women. Therefore, highest average scores were registered for somatization $(56.14 \pm 10.17)$ and compulsion $(55.02 \pm 10.11)$.

Predominant male self-identification was more common (54\%) by a small margin. For some models, highly influential observations with extreme values for one of the included predictors were detected and these models were rerun with capped extreme values. A graphical presentation of highly influential observations by CD for each model can be found in see online suppl. Figure 1; see www.karger.com/doi/10.1159/000514484 for all online suppl. material. A full list of comparisons of $t$ and $p$ values for original and capped models is provided in online suppl. Table 1.
Insulin Function and Anthropometric Parameters

Models for insulin function parameters and BMI revealed interaction effects for BMI and HOMA-IR on BSI subscore paranoia $(t=2.462, p=0.0189)$ and compulsion $(t=2.612, p=0.0133$; n.s. after capping of extreme values), as well as the total BSI score $(t=2.157, p=0.0379)$. Therefore, psychological symptom scores were rising with BMI, however only in women with likely insulin resistance indicated by a HOMA-IR score above 2.5 (see Fig. 1).

BODI 2 was associated with good insulin sensitivity, expressed by high Matsuda Index $(t=2.592, p=0.0146)$ and OGIS. For the latter, an interaction effect with BMI was found $(t=-2.405, p=0.0236)$, implicating lower burnout scores with rising insulin sensitivity only in overweight and obese women (see Fig. 1). (Also refer to Table 2 , section a).

Higher BMI was associated with female self-identification $(t=3.729, p=0.0007)$. Similarly, the FLI was associated with female self-identification $(t=3.451, p=0.0013)$.

Higher WHR was associated with higher PSSI score $(t=2.319, p=0.0297)$, interpersonal sensibility subscore of the BSI $(t=2.125, p=0.040)$, and male self-identification $(z=2.333, p=0.0196)$. Lean mass was associated with BODI $2(t=2.829, p=0.0095)$, while body fat was inversely associated $(t=-2.174, p=0.0402)$ with BODI 2. Body fat was further associated with neutral $(t=2.114$, $p=0.0436$; n.s. after capping of extreme values) and female $(t=2.865, p=0.0078)$ gender self-identification. A 2 -way interaction effect between body fat and gender selfidentification was present for the anxiety subscore of the BSI ( $t=-2.296, p=0.0299)$, while interaction effects between lean mass and gender self-identification emerged for the somatization subscores of BSI $(t=-2.948, p=$ 0.0068). (See also Fig. 2 for a graphic depiction of effects of anthropometric parameters.) A summary of the GLM results is provided in Table 2 , section $b$.

\section{Hormones}

Models for TSH, LH, FSH, testosterone, and estradiol revealed interaction effects for gender role and TSH with BODI items $2(t=-2.42, p=0.022)$ and $4(t=-2.39, p=$ $0.023)$ as well as self-oriented stress $(t=-2.11, p=0.042 ;$ n.s. after capping of extreme values). Thereby, gender self-identification showed opposite effects on all 3 parameters in dependence of TSH. While in subjects with high male selfidentification, higher TSH was associated with lower scores of BODI 2 and 4 and self-oriented stress, and in subjects with high female self-identification scores, BODI 2 and 4 were rising with TSH. Finally, there was an interaction ef- 
Table 2. GLM results

\begin{tabular}{|c|c|c|c|c|c|}
\hline \multicolumn{6}{|c|}{ (a) Models for metabolic parameters } \\
\hline BODI 2 & Matsuda Index & $33 / 30$ & 2.592 & 0.0146 & HOMA-IR, BMI, age, gender role \\
\hline Stress (work) & & $34 / 31$ & 2.569 & 0.0152 & \\
\hline Compulsion (BSI) & HOMA-IR $\times$ BMI & $39 / 34$ & 2.612 & 0.0133 & Matsuda index, age, gender role \\
\hline Female self-identification & BMI & $39 / 35$ & 3.729 & 0.0007 & HOMA-IR, Matsuda Index, gender role, age \\
\hline BODI 2 & OGIS $\times$ BMI & $29 / 24$ & -2.405 & 0.0236 & HOMA-IR, gender role, age \\
\hline Female self-identification & FLI & $42 / 39$ & 3.451 & 0.0013 & Gender role, age \\
\hline \multicolumn{6}{|c|}{ (b) Models for anthropometric parameters } \\
\hline BODI 2 & Body fat & $26 / 23$ & -2.174 & 0.0402 & Age, lean mass, gender role \\
\hline Anxiety (BSI) & Body fat $\times$ gender role & $30 / 24$ & -2.296 & 0.0299 & Age, lean mass \\
\hline & WHR & $26 / 23$ & 2.319 & 0.0297 & Age, gender role \\
\hline Interpersonal sensitivity (BSI) & & $42 / 39$ & 2.125 & 0.04 & \\
\hline \multicolumn{6}{|c|}{ (c) Models for hormone parameters } \\
\hline BODI 2 & $\mathrm{TSH} \times$ gender role & $36 / 28$ & -2.344 & 0.0257 & Age, estradiol, LH, testosterone, FSH \\
\hline BODI 4 & & $36 / 28$ & -2.481 & 0.0187 & \\
\hline Stress (self- oriented) & & $38 / 30$ & -2.112 & 0.0424 & \\
\hline Aggression (BSI) & $\mathrm{LH}$ & $42 / 35$ & -2.139 & 0.0389 & Estradiol, testosterone, TSH, FSH, age, gender role \\
\hline Aggression (BSI) & FSH & $42 / 35$ & 2.234 & 0.0314 & Estradiol, testosterone, TSH, LH, age, gender role \\
\hline Male self-identification & $\mathrm{LH} \times \mathrm{FSH}$ & $42 / 36$ & -2.231 & 0.0317 & Estradiol, testosterone, TSH, FSH, age \\
\hline Paranoia (BSI) & Cortisol (sr.) & $40 / 35$ & 2.487 & 0.0172 & Age, CRP, gender role \\
\hline Female self-identification & CRP & $42 / 37$ & 2.576 & 0.0137 & Age, cortisol, gender role \\
\hline \multicolumn{6}{|c|}{ (e) Models for biofeedback parameters } \\
\hline BODI 2 & Baseline HRV $\times$ stress HRV & $35 / 30$ & 2.399 & 0.0227 & Age, gender role, relaxation HRV \\
\hline BODI 4 & & $35 / 30$ & 2.868 & 0.0074 & \\
\hline Stress (work life) & & $35 / 30$ & 2.071 & 0.0465 & \\
\hline Psychoticism (BSI) & & & -2.870 & 0.0067 & \\
\hline Stress (family life) & Stress HRV & $37 / 33$ & -2.676 & 0.0114 & Age, gender role, baseline and relaxation HRV \\
\hline
\end{tabular}

Only significant results $(p<0.05)$ are displayed. Interaction effects are indicated by “x." FLI, fatty liver index; WHR, waist-to-hip ratio; BSI, Brief Symptom Inventory; BODI, Burnout Dimensions Inventory; PSS, Perceived Stress Scale; HRV, heart rate variability; TSH, thyroid-stimulating hormone; CRP, C-reactive protein; HOMA-IR, homeostasis model was used for assessment of insulin resistance; OGIS, oral glucose insulin sensitivity; FSH, follicle-stimulating hormone; LH, luteinizing hormone; GLM, generalized linear model.

Psychometric and Biological Correlates of Stress, Obesity, and Gender Roles 

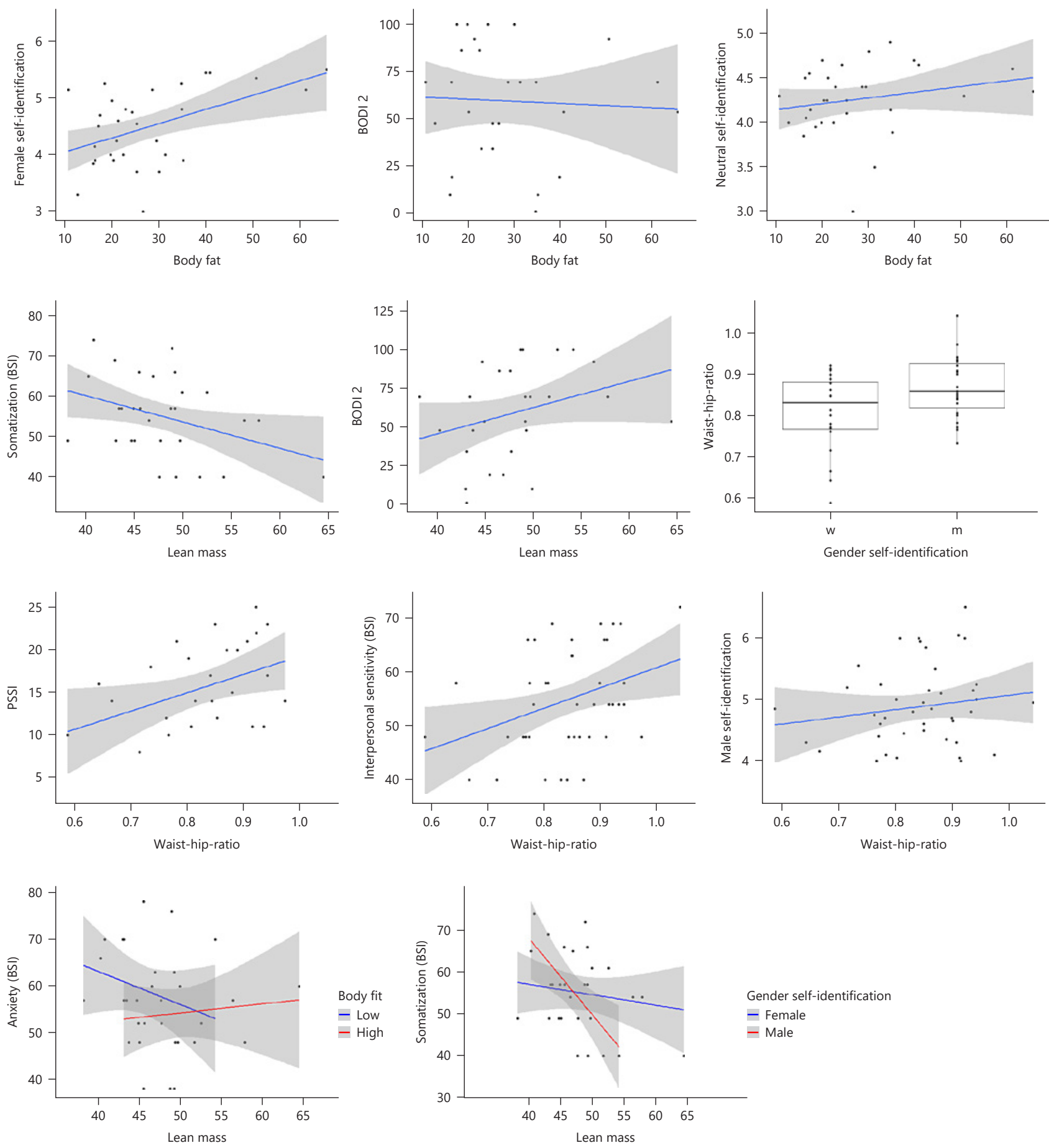

Fig. 2. Main and interaction effects from the GLMs for anthropometric parameters. Only significant $(p<0.05)$ effects are depicted. For illustration of interaction effects of 2 continuous variables, body fat was divided into a factor with 3 levels by sample distribution $( \pm 1$ SD). GLM, generalized linear model. 

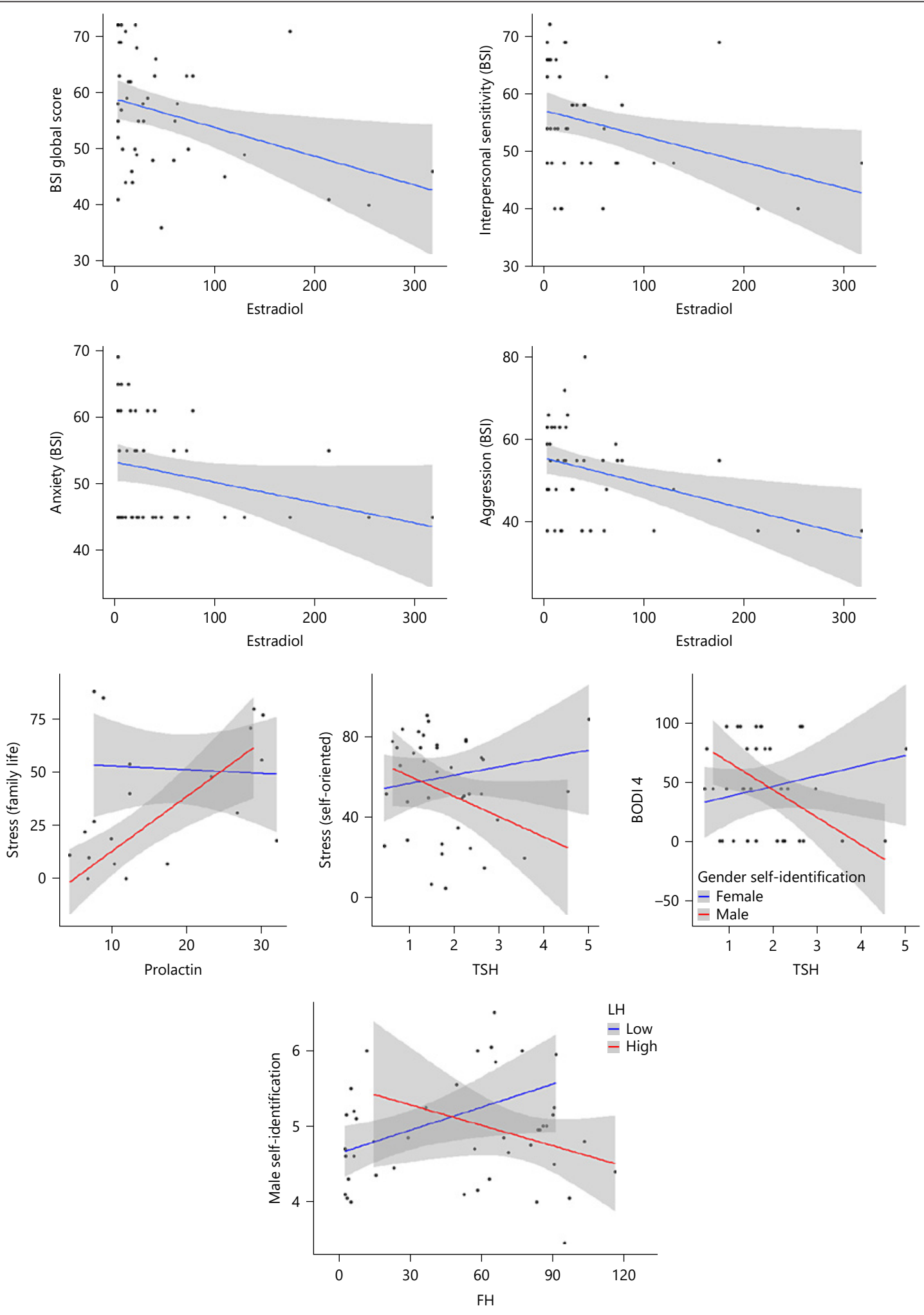

Fig. 3. Main and interaction effects from the GLMs for hormone parameters. Only significant $(p<0.05)$ effects are depicted. For illustration of interaction effects of 2 continuous variables, LH was divided into a factor with 3 levels by sample distribution ( $\pm 1 \mathrm{SD})$. GLM, generalized linear model. 


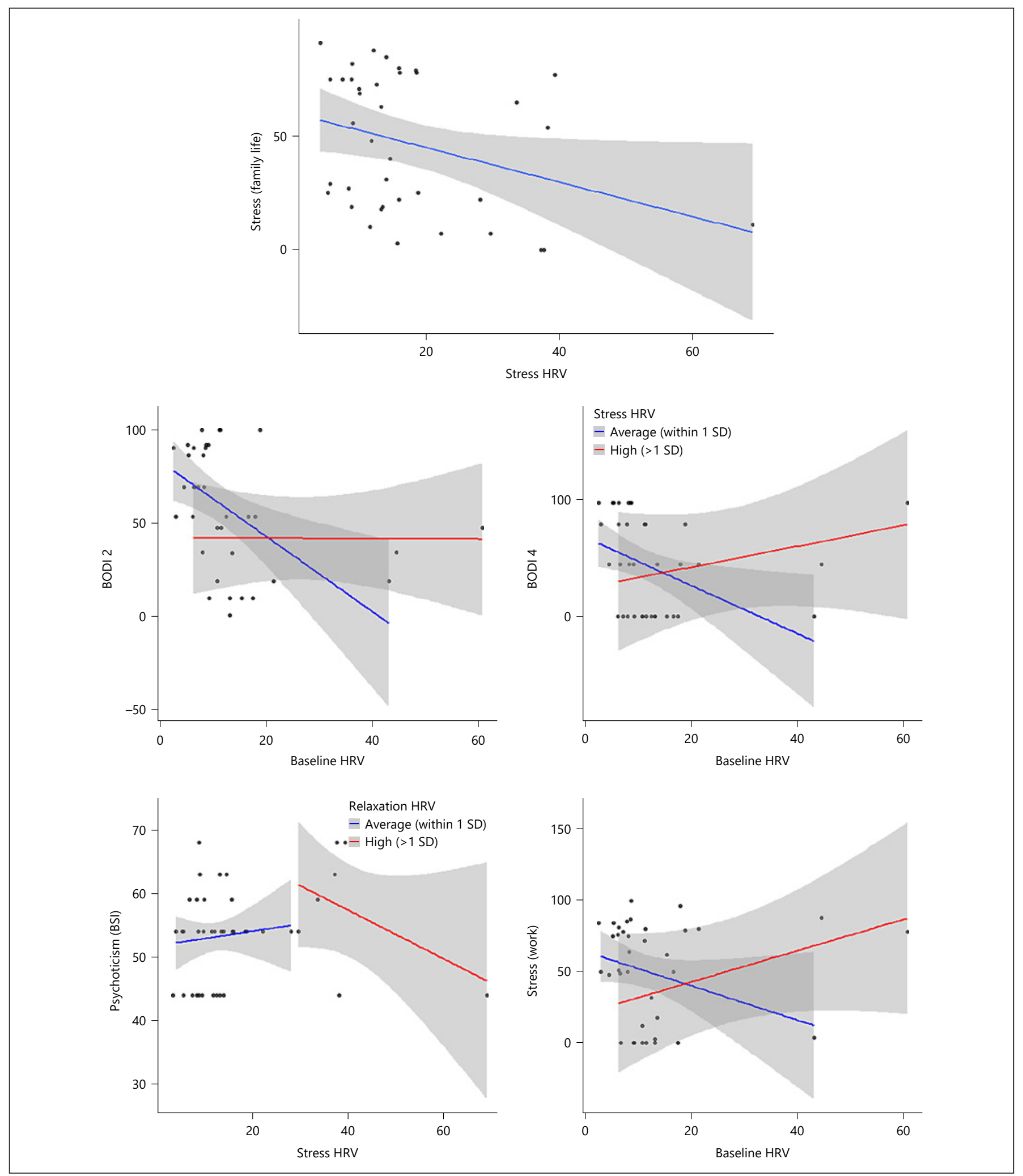

Fig. 4. Main and interaction effects from the GLMs for biofeedback parameters. Only significant $(p<0.05)$ effects are depicted. For illustration of interaction effects of 2 continuous variables, stress HRV was divided into a factor with 3 levels by sample distribution $( \pm 1$ SD). HRV, heart rate variability; GLM, generalized linear model. 
fect between LH and FSH on male self-identification ( $t=$ $-2.23, p=0.032$ ). Therefore, low levels of either FSH or LH and high levels of the other hormone were associated with male self-identification. Higher FSH $(t=2.23, p=0.031)$ and lower LH $(t=-2.14, p=0.039)$ were also associated with a higher aggression subscore of the BSI.

Higher prolactin and male self-identification were associated with family stress $(t=2.301, p=0.0352)$. Higher estradiol was associated with lower scores for BSI items interpersonal sensitivity $(t=-2.302, p=0.0268)$, aggression $(t=-3.342, p=0.0018)$, anxiety $(t=-2.273, p=$ $0.0286)$, and the global BSI score $(t=-2.629, p=0.0122)$. (See also Fig. 3 for a graphic depiction of hormone effects.) A summary of the GLM results is provided in Table 2 , section c.

\section{Inflammation and Immunology}

Models for stress parameters revealed a positive association between serum cortisol and paranoia subscores $(t=2.487, p=0.0172)$, as well as an interaction effect between gender role and sputum cortisol on BODI 2. Further analysis indicated that higher sputum cortisol was associated with higher BODI 2 , but only in subjects with predominant male self-identification $(t=2.487, p=0.0181)$. Higher CRP was associated with female self-identification $(t=$ $2.576, p=0.0137$ ). (See also Fig. 1 for a graphic depiction of effects of inflammation markers.) A summary of the GLM results is provided in Table 2, section $\mathrm{d}$.

\section{Biofeedback}

Higher stress HRV was associated with lower stress scores for family life stress $(t=-2.676, p=0.0114)$. Interaction effects were found between baseline and stress HRV on BODI items $2(t=2.399, p=0.0227)$ and $4(t=$ $2.868, p=0.0073)$ as well as stress in work life $(t=2.071$, $p=0.0465)$ and the psychoticism subscore of the BSI $(t=$ $-2.87, p=0.0067)$. Thereby, in presence of high stress HRV, lower scores of the unfavorable outcome parameters were observed, which showed a marginal impact by baseline HRV. However, in case of low stress HRV, protective effects of higher baseline HRV were present (Fig. 2).

Results on biofeedback parameters showed the greatest dependence on influential observations and changed considerably after capping of extreme values, rendering insignificant results, except for the association with the psychoticism subscore of the BSI $(t=-2.935, p=0.0057)$. (See also Fig. 4 for a graphic depiction of hormone effects.) A summary of the GLM results is provided in Table 2 , section e.

Psychometric and Biological Correlates of Stress, Obesity, and Gender Roles

\section{Discussion}

This exploratory analysis of 43 mostly overweight and obese women with self-perceived stress further linked immuno-metabolic parameters as well as gender roles to psychometric outcomes. Therefore, a mediating effect of gender self-identification on several associations could be observed. Female self-identification most importantly correlated with a higher BMI, standing out as the most robust finding $(p=0.0007)$. Along these lines, female self-identification was also associated with higher body fat, FLI, and CRP. Chronic subclinical inflammation is a well-published finding in obesity, putatively due to an inadequate supply of oxygenation in excessive body fat [32]. Obesity also states a potential substrate for mental and metabolic disorders [33, 34]. Here, we present a first link to gender self-identification. Male gender self-identification, on the other hand, was associated with family stress and higher burnout scores of impaired distancing ability in interplay with elevated sputum cortisol. Among inflammation markers, a singular main effect of serum cortisol was found for the paranoia but not depression or anxiety subscores of the BSI, indicating a special vulnerability of psychosis spectrum symptoms to stress. It is a well-known finding that psychotic episodes are often anteceded by increased psychological stress, and in agreement with this, a stressreactive rise in paranoia was also demonstrated before in healthy subjects $[35,36]$.

Further, women with higher male self-identification showed higher WHR, thereby mirroring the WHR attributed to male physics. Interestingly, higher WHR was further associated with higher perceived stress and interpersonal sensitivity. Despite some inconsistency, the WHR was previously identified as a predictor of sequelae of metabolic syndrome, such as cardiovascular risk, in a sexand BMI-specific manner [37, 38]. These results, on the one hand, may implicate that known gender differences in social and emotional bonding and unfavorable stress and help-seeking behavior attributed to male sex are rather related to gender roles than biological sex $[39,40]$. On the other hand, a mismatch between anthropometric societal norms and women's actual physics may lead to insecurity and stress, which are known risk factors for cardiovascular events [41]. To that effect, the burnout parameter of reduced distancing was rising with body fat, whereas lean body mass showed a protective effect. Similarly, the somatization and anxiety subscores of the BSI were affected by body fat and lean body mass. A higher $\mathrm{BMI}$ in women with higher state anxiety was observed in

Neuroendocrinology 2022;112:130-142 DOI: $10.1159 / 000514484$ 
some but not all studies, and the direction of this association remains unresolved so far $[42,43]$.

Higher levels of estrogen were associated with lower BSI global score but also subscores for interpersonal sensitivity, aggression, and anxiety. Thus, a hormonal contribution to BSI subscores is suggested, thereby strengthening previous findings of protective effects of estrogen for mental health [44]. Surprisingly, the depression subscore of the BSI did not show any associations, but rather subscores attributable to gender role norms and stereotypes, such as interpersonal sensitivity, anxiety, and aggression. Low FSH and high $\mathrm{LH}$, a pattern also characteristic for polycystic ovary syndrome, were associated with high aggression subscores and male self-identification. Aggression is a typical symptom of some personality disorders and ADHD, both of which were associated with elevated male sex hormones and polycystic ovary syndrome $[45,46]$. Male gender role self-identification, however, was also higher in women with low LH and high FSH. A mismatch in sexual hormones may therefore affect gender roles. In summary, these findings advocate a multifactorial model for sex and gender effects on psychometabolic parameters. This is underlined also by the more complex pattern of TSH effects on burnout scores, that, based on our preliminary results, may be oppositional in dependency on predominant gender self-identification. This may be related to allostatic adaptations of thyroid hormone trafficking in response to dietary changes and stress load [47].

Concerning biological stress response, differences in stress, and burnout parameters were observed in dependency of HRV. Flexibility in heart rate is thereby believed to mirror an organism's capability to react adequately and specifically to different external stimuli and is putatively reduced in patients suffering from anxiety disorders. In full agreement with earlier work [48], higher baseline and stress HRV showed protective effects, here on family and job stress as well as BODI items reduced ability of distancing and dysfunctional compensation. These results further strengthen the role of HRV in stress response, which may be linked to central nervous stress processing in the medial prefrontal cortex according to a growing body of neuroimaging studies [49].

Despite $9 \%$ of the women being in a prediabetic state, $\beta$-cell function and insulin secretion were intact in this sample and no associations were found for these parameters. For OGIS, a moderating effect of BMI on insulin sensitivity was found. Despite not reaching statistical significance, this pattern could also be observed for the Matsuda Index. Hereby, women with a BMI above 25 showed a negative correlation of BODI 2 and insulin sensitivity. Following previous reports of impaired insulin sensitivity in burnout patients, this result was to be expected [50]. However, women with normal BMI rather showed a reduction in ability of distancing with higher insulin sensitivity. This peculiar finding may be related to the concept of glucose brain pull described by the selfish brain hypothesis. Accordingly, chronic stress may lead to a flattened stress response that causes a shift in glucose trafficking from the brain to the body and subsequent weight gain. Increased insulin sensitivity in women with higher burnout scores but normal body weight may depict an earlier stage within this concept in which stress leads to a compensational increase in insulin sensitivity that may be followed by a shift with weight gain and insulin resistance once the stress load becomes protracted. Nevertheless, this finding contrasts well-established basic as well as clinical research, implying that stress quickly impairs insulin sensitivity. It may be owed to the very low rate of clinically relevant impaired insulin sensitivity (Matsuda Index of below 2), as well as the small sample size and the exploratory nature of this study. Likewise, the interaction effects of HOMA-IR and BMI on the global BSI score as well as compulsion and paranoia do agree with the current body of evidence, indicating unfavorable psychological outcomes in obese subjects at risk for insulin resistance. A mediating effect of BMI on stress response was shown before and may be related to the endocrine functions of body fat [51].

The scope of these findings is limited by the exploratory nature of the analyses. While most findings agree with and expand on previous literature, due to the small size of our sample and the broad application of tests, false-positive findings are to be expected. Results therefore must be interpreted cautiously until they will be clarified in a larger sample or a more focused replication analysis.

Further, there may be a selection bias as women interested in stress- and weight reduction visiting the "la pura" women's health resort were recruited, hence resulting in a sample that may show socioeconomic and other differences compared to the general population. Despite thorough clinical characterization of the women recruited for this study, including an assessment by a trained clinical psychologist, no psychiatric diagnostic interview was conducted. Therefore, the presence of a manifest psychiatric diagnosis that was suggested by the BSI results in some women cannot be ruled out nor confirmed. Finally, women showed a wide range of age, anthropometric and psychometric parameters. Less stringent recruitment criteria lead to a more naturalistic sample, better portraying real-life circumstances, but bear a risk for a degree of het- 
erogeneity too big for the sample size at hand. Along these lines, some results may be driven primarily by few, highly influential patients. To deal with this limitation, we checked for extreme values and reran relevant models with capped extreme values, mostly confirming the results of the original models. Finally, we did not consider pre- or postmenopausal state in this analysis due to the limited sample size. The majority of women (79\%) was in a peri- or postmenopausal state, and results especially on sexual hormones may only be relevant in this context.

In synopsis, we present several lines of evidence linking female self-identification to obesity and corresponding unfavorable metabolic and immunological markers. Obesity has already been an urgent call for global stakeholders and practitioners and may be even more relevant in light of COVID-19, bringing along restrictions on physical activity and changing consumption behavior to the worse [52]. Links between obesity and psychosocial well-being were already drawn and recently strengthened by research on COVID-19, especially in women. A correlation of female self-identification with BMI was the most robust finding in this analysis; however, the direction of the association remains unresolved due to the cross-sectional nature. Female self-identification bringing along a lifestyle more associated with weight gain is contrasted by a higher chance to pick up a female gender role in women with higher BMI. Either way, the association with CRP and a higher likelihood of fatty liver disease suggests relevant clinical risk in women with predominant female gender roles. Incorporating gender roles, instead of just biological sex, into clinical evaluation paradigms may therefore better capture metabolic risk and help to close in on precision medicine in neuroendocrinology.

\section{Acknowledgements}

We are thankful to the patients for enabling this study and all personnel of the Medical University of Vienna and the "la pura" women's health resort who assisted the study team.

\section{Statement of Ethics}

All study-related procedures were approved by the responsible Ethics Committee, "Ethikkomission Niederösterreich" (GS1EK-4/517-2017). All subjects gave written consent to partaking in the study after thorough oral and written education about the study. All research was conducted ethically in accordance with the World Medical Association Declaration of Helsinki.

\section{Conflict of Interest Statement}

The authors have no conflicts of interest to declare.

\section{Funding Sources}

There are no funding sources for the presented research.

\section{Author Contributions}

Dr. A. Kautzky performed all data analyses and was involved in study planning. Mag. Kathrin Heneis was responsible for all psychometric assessments. Dr. K. Stengg and Dr. S. Fröhlich were responsible for clinical assessment and supervision of all subjects. Prof. Dr. A. Kautzky-Willer was responsible for study planning and supervision of all study-related procedures.

\section{References}

1 Danaei G, Finucane MM, Lu Y, Singh GM, Cowan MJ, Paciorek CJ, et al. National, regional, and global trends in fasting plasma glucose and diabetes prevalence since 1980: systematic analysis of health examination surveys and epidemiological studies with 370 country-years and 2.7 million participants. Lancet. 2011 Jul 2;378(9785):31-40.

2 GBD 2015 Disease and Injury Incidence and Prevalence Collaborators. Global, regional, and national incidence, prevalence, and years lived with disability for 310 diseases and injuries, 1990-2015: a systematic analysis for the Global Burden of Disease Study 2015. Lancet. 2016 Oct 8;388(10053):1545-602.

3 Whiteford HA, Ferrari AJ, Degenhardt L, Feigin $\mathrm{V}$, Vos T. The global burden of mental, neurological and substance use disorders: an analysis from the Global Burden of Disease Study 2010. PLoS One. 2015;10(2):e0116820.

4 Chooi YC, Ding C, Magkos F. The epidemiology of obesity. Metabolism. 2019 Mar;92:610.

5 Gregg EW, Sattar N, Ali MK. The changing face of diabetes complications. Lancet Diabetes Endocrinol. 2016 Jun;4(6):537-47.

6 Saeedi P, Petersohn I, Salpea P, Malanda B, Karuranga S, Unwin N, et al. Global and regional diabetes prevalence estimates for 2019 and projections for 2030 and 2045: results from the International Diabetes Federation Diabetes Atlas, 9(th) edition. Diabetes Res Clin Pract. 2019 Nov; 157:107843.

7 Keyloun KR, Hansen RN, Hepp Z, Gillard P, Thase ME, Devine EB. Adherence and persistence across antidepressant therapeutic class- es: a retrospective claims analysis among insured US patients with major depressive disorder (MDD). CNS Drugs. 2017 May;31(5): 421-32.

8 McGovern A, Tippu Z, Hinton W, Munro N, Whyte $\mathrm{M}$, de Lusignan S. Comparison of medication adherence and persistence in type 2 diabetes: a systematic review and meta-analysis. Diabetes Obes Metab. 2018 Apr;20(4): 1040-3.

9 Darwish L, Beroncal E, Sison MV, Swardfager $\mathrm{W}$. Depression in people with type 2 diabetes: current perspectives. Diabetes Metab Syndr Obes. 2018;11:333-43.

10 Hackett RA, Steptoe A. Type 2 diabetes mellitus and psychological stress: a modifiable risk factor. Nat Rev Endocrinol. 2017 Sep; 13(9):547-60. 
11 Joseph JJ, Golden SH. Cortisol dysregulation: the bidirectional link between stress, depression, and type 2 diabetes mellitus. Ann N Y Acad Sci. 2017 Mar;1391(1):20-34.

12 Lontchi-Yimagou E, Sobngwi E, Matsha TE, Kengne AP. Diabetes mellitus and inflammation. Curr Diab Rep. 2013 Jun;13(3):435-44.

13 Goldsmith DR, Rapaport MH, Miller BJ. A meta-analysis of blood cytokine network alterations in psychiatric patients: comparisons between schizophrenia, bipolar disorder and depression. Mol Psychiatry. 2016 Dec;21(12): 1696-709.

14 Goessl VC, Curtiss JE, Hofmann SG. The effect of heart rate variability biofeedback training on stress and anxiety: a meta-analysis. Psychol Med. 2017 Nov;47(15):2578-86.

15 Sessa F, Anna V, Messina G, Cibelli G, Monda V, Marsala G, et al. Heart rate variability as predictive factor for sudden cardiac death. Aging. 2018 Feb 23;10(2):166-77.

16 Goldenberg I, Goldkorn R, Shlomo N, Einhorn M, Levitan J, Kuperstein R, et al. Heart rate variability for risk assessment of myocardial ischemia in patients without known coronary artery disease: the HRV-DETECT (Heart Rate Variability for the Detection of Myocardial Ischemia) Study. J Am Heart Assoc. 2019 Dec 17;8(24):e014540.

17 van der Zwan JE, de Vente W, Huizink AC, Bögels SM, de Bruin EI. Physical activity, mindfulness meditation, or heart rate variability biofeedback for stress reduction: a randomized controlled trial. Appl Psychophysiol Biofeedback. 2015 Dec;40(4):257-68.

18 Lehrer P, Kaur K, Sharma A, Shah K, Huseby $\mathrm{R}$, Bhavsar J, et al. Heart rate variability biofeedback improves emotional and physical health and performance: a systematic review and meta analysis. Appl Psychophysiol Biofeedback. 2020 Sep;45(3):109-29.

19 Van de Velde S, Boyd A, Villagut G, Alonso J, Bruffaerts R, De Graaf R, et al. Gender differences in common mental disorders: a comparison of social risk factors across four European welfare regimes. Eur J Public Health. 2019 Jun 1;29(3):481-7.

20 Matthews DR, Hosker JP, Rudenski AS, Naylor BA, Treacher DF, Turner RC. Homeostasis model assessment: insulin resistance and beta-cell function from fasting plasma glucose and insulin concentrations in man. Diabetologia. $1985 \mathrm{Jul} ; 28(7): 412-9$.

21 Matsuda M, DeFronzo RA. Insulin sensitivity indices obtained from oral glucose tolerance testing: comparison with the euglycemic insulin clamp. Diabetes Care. 1999 Sep;22(9): 1462-70.

22 DeFronzo RA, Matsuda M. Reduced time points to calculate the composite index. Diabetes Care. 2010 Jul;33(7):e93.

23 Goedecke JH, Dave JA, Faulenbach MV, Utzschneider KM, Lambert EV, West S, et al. Insulin response in relation to insulin sensitivity: an appropriate beta-cell response in black South African women. Diabetes Care. 2009 May;32(5):860-5.

24 Bergman RN, Ader M, Huecking K, Van Citters G. Accurate assessment of beta-cell function: the hyperbolic correction. Diabetes. 2002 Feb;51(Suppl 1):S212-20.

25 Bedogni G, Bellentani S, Miglioli L, Masutti F, Passalacqua M, Castiglione A, et al. The Fatty Liver Index: a simple and accurate predictor of hepatic steatosis in the general population. BMC Gastroenterol. 2006 Nov 2;6:33.

26 Derogatis LR. Brief symptom inventory (BSI): administration, scoring; and procedures manual. Minneapolis, MN: National Computer Systems; 1993.

27 Scheibenbogen O, Andorfer U, Kuderer M, Musalek M. Prävalenz des Burnout-Syndroms in Österreich. Abschlussbericht Bundesministeriums für Arbeit, Soziales und Konsumentenschutz (BMASK); 2017.

28 Cohen S, Kamarck T, Mermelstein R. A global measure of perceived stress. J Health Soc Behav. 1983;24(4):385-96.

29 Bem D, Lipsitz S. Bem sex-role inventory. Mental measurements yearbook with tests in print. 1981. Vol. 9.

30 Mintu KD, Gogoi B. Influential observations and cutoffs of different influence measures in multiple linear regression. Int J Comp Theo Stat. 2015;2(2):7.

31 Alberti G, Zimmet P, Shaw J. IDF Consensus worldwide definition of the metabolic syndrome. International Diabetes Federation; 2006.

32 Karczewski J, Śledzińska E, Baturo A, Jończyk I, Maleszko A, Samborski P, et al. Obesity and inflammation. Eur Cytokine Netw. 2018 Sep 1;29(3):83-94.

33 Halim M, Halim A. The effects of inflammation, aging and oxidative stress on the pathogenesis of diabetes mellitus (type 2 diabetes). Diabetes Metab Syndr. 2019 Mar-Apr;13(2): 1165-72.

34 Herder C, Hermanns N. Subclinical inflammation and depressive symptoms in patients with type 1 and type 2 diabetes. Semin Immunopathol. 2019 Jul;41(4):477-89.

35 Lincoln TM, Peter N, Schäfer M, Moritz S. Impact of stress on paranoia: an experimental investigation of moderators and mediators. Psychol Med. 2009 Jul;39(7):1129-39.

36 Moritz S, Burnette P, Sperber S, Köther U, Hagemann-Goebel M, Hartmann M, et al. Elucidating the black box from stress to paranoia. Schizophr Bull. 2011 Nov;37(6):1311-7.

37 Li C, Engström G, Hedblad B, Calling S, Berglund G, Janzon L. Sex differences in the relationships between BMI, WHR and incidence of cardiovascular disease: a population-based cohort study. Int J Obes. 2006 Dec;30(12): 1775-81.

38 Cao Q, Yu S, Xiong W, Li Y, Li H, Li J, et al. Waist-hip ratio as a predictor of myocardial infarction risk: a systematic review and metaanalysis. Medicine. 2018 Jul;97(30):e11639.
39 Fiori KL, Denckla CA. Social support and mental health in middle-aged men and women: a multidimensional approach. J Aging Health. 2012 Apr;24(3):407-38.

40 McKenzie SK, Collings S, Jenkin G, River J. Masculinity, social connectedness, and mental health: men's diverse patterns of practice. Am J Mens Health. 2018 Sep;12(5):1247-61.

41 Afrisham R, Paknejad M, Soliemanifar O, Sadegh-Nejadi S, Meshkani R, Ashtary-Larky D. The influence of psychological stress on the initiation and progression of diabetes and cancer. Int J Endocrinol Metab. 2019 Apr; 17(2): e67400.

42 Mestre ZL, Melhorn SJ, Askren MK, Tyagi V, Gatenby C, Young L, et al. Effects of anxiety on caloric intake and satiety-related brain activation in women and men. Psychosom Med. 2016 May;78(4):454-64.

43 Stanikova D, Luck T, Pabst A, Bae YJ, Hinz A, Glaesmer $\mathrm{H}$, et al. Associations between anxiety, body mass index, and sex hormones in women. Front Psychiatry. 2019;10:479.

44 Albert KM, Newhouse PA. Estrogen, stress, and depression: cognitive and biological interactions. Annu Rev Clin Psychol. 2019 May 7;15:399-423.

45 Scaruffi E, Gambineri A, Cattaneo S, Turra J, Vettor R, Mioni R. Personality and psychiatric disorders in women affected by polycystic ovary syndrome. Front Endocrinol. 2014;5:185.

46 Rodriguez-Paris D, Remlinger-Molenda A, Kurzawa R, Głowińska A, Spaczyński R, Rybakowski $\mathrm{F}$, et al. Psychiatric disorders in women with polycystic ovary syndrome. Psychiatr Pol. 2019 Aug 31;53(4):955-66.

47 Chatzitomaris A, Hoermann R, Midgley JE, Hering S, Urban A, Dietrich B, et al. Thyroid allostasis-adaptive responses of thyrotropic feedback control to conditions of strain, stress, and developmental programming. Front Endocrinol. 2017;8:163.

48 Jarvelin-Pasanen S, Sinikallio S, Tarvainen MP. Heart rate variability and occupational stress-systematic review. Ind Health. 2018 Nov 21;56(6):500-11.

49 Kim HG, Cheon EJ, Bai DS, Lee YH, Koo BH. Stress and heart rate variability: a meta-analysis and review of the literature. Psychiatry Investig. 2018 Mar;15(3):235-45.

50 Fernandez-Montero A, García-Ros D, Sánchez-Tainta A, Rodriguez-Mourille A, Vela A, Kales SN. Burnout syndrome and increased insulin resistance. J Occup Environ Med. 2019 Sep;61(9):729-34.

51 Ozaslan B, Patek SD, Grabman JH, Shepard JA, Dassau E, Breton MD, et al. Body mass index effect on differing responses to psychological stress in blood glucose dynamics in patients with type 1 diabetes. J Diabetes Sci Technol. 2018 May;12(3):657-64.

52 Khan MA, Moverley Smith JE. "Covibesity," a new pandemic. Obes Med. 2020 Sep; 19: 100282 . 